\title{
La Propiedad Intelectual y desarrollo: Problemas para la capitalización de activos intangibles
}

\author{
Intellectual Property and development: Problems for \\ the capitalization of intangible assets
}

\author{
René Antonio Gálvez Delgado \\ Investigador jurídico independiente \\ Ciudad: Quito \\ País: Ecuador
}

Artículo Original (Nota de investigación)

RFJ, No. 7, 2020, pp. 406-424, ISSN 2588-0837

RESUMEN: En el presente artículo se abordan cuestiones referentes a la gestión de la Propiedad Intelectual desde la legislación y la importancia que este campo de la ciencia jurídica tiene en la esfera del desarrollo social contemporáneo, además se abordan temáticas referentes a la necesidad de implementar mecanismos para optimizar la capitalización de activos intangibles de Propiedad Intelectual y permitir el pleno goce de sus frutos por su titular. En las siguientes líneas se mencionarán algunos aspectos importantes a ser considerados para que la gestión de derechos de Propiedad Intelectual en el Ecuador sea eficiente, con bajos costos de transacción y que refleje las necesidades de la cuarta revolución industrial y el mercado global de la actualidad.

PALABRAS CLAVE: Propiedad intelectual, desarrollo económico, eficiencia, economía, regulación.

ABSTRACT: This research article addresses issues related to the management of Intellectual Property from the legislation and the importance that this field of legal studies has in the sphere of contemporary social development, as well as topics related to the need to implement mechanisms to optimize the capitalization of intangible assets of Intellectual Property and allow the full enjoyment of its benefits by its owners. Then, the 
following lines will mention some crucial aspects that positively impact the management of Intellectual Property rights in Ecuador. It means improving efficiency, lowering transactional costs, and reflecting the needs of the fourth industrial revolution and the current global market.

KEYWORDS: Intellectual property, economic development, efficiency, economy, regulation.

\section{INTRODUCCIÓN}

A través de la historia el ser humano ha ocupado su intelecto para encontrar mecanismos capaces de superar las dificultades que se presentan en el camino del desarrollo y precisamente el producto de aquella búsqueda es lo que hoy conocemos como tecnología que se constituye en un conjunto de creaciones y conocimientos elaborados y perfeccionados a lo largo del tiempo y que están destinados a resolver problemas de la vida cotidiana.

De esta manera, la Propiedad Intelectual aparece como un campo de la ciencia jurídica destinado a proteger a las personas que han plasmado su capacidad inventiva e ingenio en algún mecanismo capaz de mejorar la vida de quienes integran la sociedad, sin embargo, una segunda dimensión de la propiedad intelectual, como ente creador de riqueza, ha sido ignorada de forma generalizada.

En este ensayo se procederá a analizar esta segunda dimensión, su importancia y algunos de los principales problemas de la legislación ecuatoriana, tanto generales como específicos, que impiden capitalizar los beneficios de la propiedad intelectual y generar desarrollo.

\section{EL ROL DE LA PROPIEDAD INTELECTUAL EN EL DESARROLLO HUMANO.}

Sin lugar a dudas el ser humano ha aprovechado su gran intelecto para convertirse en la especie dominante del planeta y esto ha puesto a su disposición un sinnúmero de posibilidades y recursos para trascender sus límites, ha cumplido metas que 
se veían como insuperables hace tan solo unos pocos años a consecuencia de sus diversas limitaciones físicas.

El homo sapiens ha logrado llegar al espacio y caminar sobre el satélite natural de su planeta, ha conseguido cartografiar las profundidades del océano a precisión y en la actualidad incluso mantiene bajo control varias patologías potencialmente mortales por lo que su expectativa de vida ha aumentado considerablemente.

Estos logros no han sido gratuitos, cada uno de ellos ha demandado la inversión de tiempo y recursos en proporciones descomunales por parte de personas comunes y corrientes que únicamente concibieron una idea y tuvieron la convicción que esta contaba con la posibilidad de transformar la realidad de la sociedad para siempre.

De esta forma, en algún punto de la historia nace la Propiedad Intelectual como una respuesta a la necesidad de proteger y reconocer a aquellas personas que han sufrido sacrificios para mejorar nuestras condiciones como especie (Robayo, 2017, p. 63).

A partir de la propiedad intelectual se derivan los bienes inmateriales que tienen su origen en investigaciones, invenciones y creaciones resultantes de la actividad intelectual y dichos bienes encuentran protección en esta clase especial de propiedad que concede un beneficio real o potencia a su titular (Londoño \& Restrepo, 2013, p. 27).

En tal sentido, se puede afirmar que la propiedad intelectual es una especie de aliciente que se otorga a aquellas personas que han dedicado tiempo y recursos en solucionar problemas con los que la sociedad tiene debe lidiar y quienes son reconocidos con ella tienen la facultad de explotar de forma exclusiva sus creaciones con el objetivo de obtener algún beneficio económico (Rozanski, 2003, p. 106).

La Propiedad Intelectual, en su dimensión de incentivo para el creador, es una herramienta con la que el tiene la posibilidad de obtener beneficios económicos para sí al mismo tiempo que aporta al desarrollo social, dicho en otras palabras, 
el titular capitaliza el producto de su intelecto lo que constituye una motivación para que continúe invirtiendo su capacidad intelectual en encontrar soluciones prácticas a problemas cotidianos.

Como se expone en la afirmación anterior, en un escenario ideal la Propiedad Intelectual constituye un incentivo para los creadores y para ello resulta de vital importancia que la regulación sea consecuente con el objetivo de permitir a sus titulares obtener una recompensa por su inversión de tiempo y recursos.

En la actualidad es posible identificar que en ciertas naciones, principalmente en países con una cultura jurídica avanzada, la Propiedad Intelectual funciona de forma correcta al generar los incentivos necesarios para que sus titulares exploten económicamente sus activos intangibles mediante la incursión en negocios que eventualmente pueden traducirse en empresas que generen recursos y plazas de empleo aportando así al desarrollo social.

Es destacable el caso de Estados Unidos y la gran mayoría de países europeos donde el sistema ha permitido que una parte importante de la economía nacional gire en torno a los activos intangibles de propiedad intelectual los cuales constituyen una importante fracción de su Producto Interno Bruto e incluso son uno de sus principales objetos de exportación.

Hoy en día la Propiedad Intelectual está ganando cada vez más terreno en las principales economías del mundo y la globalización ha aumentado el protagonismo de los activos intangibles de Propiedad Intelectual a un ritmo nunca antes visto gracias al intercambio de masivas cantidades de información en fracciones de segundo. La cuarta revolución industrial ha traído consigo un mundo globalizado donde se han potenciado los procesos de transferencia de tecnología y esto hace previsible que en un futuro cercano la importancia de la Propiedad Intelectual será aún mayor. 
La enorme importancia de los activos intangibles de Propiedad Intelectual ya se puede dimensionar, según informe Global Intangible Finance Tracker 2018 actualmente los activos intangibles ocupan un $52 \%$, del valor total de las empresas alrededor del mundo y la tendencia es que cada vez este porcentaje continúe en aumento (Diario El País, 2018).

En ese sentido, durante lasúltimas décadas las economías más grandes del mundo han tenido especial preocupación en buscar mecanismos que garanticen la protección de sus conocimientos tecnológicos y sus innovaciones, no solamente dentro sino también afuera de sus fronteras en donde, por variaciones en el régimen de gestión de Propiedad Intelectual pueden peligrar sus activos intangibles.

Uno de los instrumentos para proteger la capitalización de la Propiedad Intelectual a nivel internacional es el Acuerdo sobre los Aspectos de los Derechos de Propiedad Intelectual relacionados con el Comercio (ADPIC). Este tratado tiene un papel fundamental ya que establece parámetros mínimos de protección de activos intangibles entre los Estados miembros y proporciona una serie de herramientas encaminadas a guiar la gestión de los derechos de Propiedad Intelectual en su ámbito comercial internacional.

La importancia de los ADPIC resulta manifiesta desde el ámbito del de Derecho Internacional en donde se puede calificar a este acuerdo como una norma "hardlaw", esto significa que es de cumplimiento obligatorio y dicha fuerza vinculante tiene su origen en la presión ejercida por parte de las economías más desarrolladas a miras de proteger sus activos intangibles en otros territorios, por ejemplo, la firma de este acuerdo constituye uno de los requisitos para formar parte de la Organización Mundial de Comercio (Mutter, 2006, p. 92).

Este acuerdo constituye una viva muestra de lo que significa la Propiedad Intelectual en nuestros días para las principales economías del mundo y evidencia la relevancia de la que gozarán los activos intangibles en un futuro cercano. 
Aquellos Estados que hoy poseen las primeras economías del planeta en el pasado no tenían un modelo muy distinto al que tienen hoy los países en vías de desarrollo ya que poseían una economía basada fundamentalmente en la producción y exportación de materias primas e incluso buscaban colonizar otros territorios en busca de mayores recursos naturales que les permitan seguir con su modo de vida, sin embargo, después de la segunda guerra mundial y con la llegada del nuevo milenio su perspectiva ha cambiado por completo y le han otorgado un peso mayor al valor del conocimiento como instrumento generador de riqueza y como la base de una economía industrializada.

En tal virtud, no es coincidencia que las principales economías del mundo implementen un sistema fuerte de Propiedad Intelectual que idealizado para proteger la gran cantidad de conocimiento e innovación que manejan y que ha contribuido a su desarrollo y posicionamiento como potencias a nivel internacional como es el caso de Japón o Alemania, países en donde tan salo hace 70 años panorama no era muy distinto al que se vive hoy en día en América Latina.

El caso de los Estados Unidos es interesante ya que refleja la capitalización de activos intangibles en su máxima expresión, el cine estadounidense en sus inicios no era superior al cine mexicano, sin embargo, a través de las décadas se fue consolidando como una de las industrias con más prestigio e ingresos en el mundo entero y este éxito es consecuencia de una política de capitalización de activos intangibles muy fuerte que se puede considerar pionera en la incursión en una cultura de consumo digital antes que una cultura de consumo material (Míguez, 2018, p. 47).

Es evidente que en algún punto de la historia quienes hoy gozan del título de países desarrollados supieron aprovechar en su momento los beneficios de comercializar ciertos bienes o servicios que generen entretenimiento, recreación o tengan la capacidad de resolver algún problema o incomodidad de forma creativa.

Sin duda alguna, el futuro de la economía mundial se encontrará fuertemente ligado a los activos de Propiedad Inte- 
lectual como resultado de la revolución tecnológica que el mundo está experimentando, por ello, es necesario replantear varios conceptos que antes se consideraban inmutables para alcanzar a comprender el valor que puede tener hoy en día una marca, un diseño industrial o una patente o cualquier otro derecho derivado de la Propiedad Intelectual.

\section{PRINCIPALES PROBLEMAS DEL MARCO REGULATORIO PARA GENERAR CAPITAL Y GOZAR DE LOS DERECHOS DE PROPIEDAD INTELECTUAL.}

La dimensión de la propiedad intelectual como una herramienta capaz de producir riqueza y mejorar las condiciones de vida de una sociedad se puede ver afectada por una serie de inconvenientes que suelen ser comunes a toda nación que está en vías de desarrollo, estos problemas tienen sus raíces principalmente en las características de sus economías y de sus ordenamientos jurídicos tal y como se podrá apreciar en las siguientes líneas.

La ineficiencia de la regulación en materia de activos de Propiedad Intelectual constituye un tema al cual no se ha dedicado suficiente atención en países como el Ecuador que aún conservan una economía basada en la exportación de materias primas y con un desarrollo jurídico muy básico en estos temas con relación a otras naciones.

Este dilema, que suele ser común a los ciudadanos de América Latina se genera como consecuencia de las ineficientes prácticas jurídicas y económicas adoptadas por los países de la región los cuales han aislado a gran parte de la población a un plano en el que no pueden generar capital en sus negocios jurídicos de forma eficiente y por ende se encuentran en cierta forma fuera de los beneficios del capitalismo.

La relación de la problemática anterior con la Propiedad Intelectual es estrecha ya que a consecuencia de estos problemas las personas de a pie no encuentran los mecanismos para capitalizar el producto de su intelecto y esto se traduce en el hecho que en países de América Latina la Propiedad Intelectual no cumple con su rol de incentivo a los titulares para que conti- 
núen con su labor creativa y aporten al desarrollo.

La calidad de la legislación, pese a ser un elemento no determinante, resulta muy importante para conseguir la consolidación saludable de cualquier negocio de tal suerte que se pueda realizar en términos claros y que ante cualquier eventualidad adversa se pueda llevar el caso ante los tribunales correspondientes en pro de obtener justicia. La legislación no es perfecta, sin embargo, como parámetro mínimo esta debe contar con las condiciones de resolver problemas comunes en el tráfico jurídico.

A fin de solucionar estas deficiencias en la legislación se puede acudir a la doctrina del Análisis Económico del Derecho bajo la cual a estos problemas que genera la legislación en el tráfico jurídico se los define como como "costos de transacción" que se pueden entender como costos que se deben realizar para poder utilizar el mercado (Arias \& Caballero, 2003, p. 118).

Sin bien los costos de transacción son inevitables y estarán presentes en cada negocio jurídico, es posible reducirlos hasta un punto en el cual no representen un problema significativo para las partes y no entorpezcan el mercado.

A fin de reducir los costos de transacción presentes en el ámbito legislativo, el Análisis Económico del Derecho sugiere recurrir a una evaluación de los efectos de la norma en el sujeto a regular, dicho análisis debe involucrar un control ex ante y ex post a la aplicación de la norma en cuestión (Plata, 2007).

Para aplicar este enfoque de mejora regulatoria del Análisis Económico del Derecho resulta necesario despojarse del sesgo generalizado que suelen tener los abogados quienes tienden a pensar que la ley por si sola es eficiente, como si estuviera dotada de un efecto "Mágico" per sé.

Esta errada pre concepción genera que no se tenga una visión amplia de las consecuencias de la legislación, aumentando los costos de transacción incluso en la esfera de las relaciones contractuales en donde se pueden generar serias dificultades para concretar negocios y, por su puesto, capitalizar sus activos de Propiedad Intelectual. 
Desde la visión clásica la ley es creada con la convicción de que, el legislador que al seguir el procedimiento formal y los requisitos de validez genera una condición suficiente para garantizar la eficiencia, lo cierto es que esta concepción ignora varios elementos que solo pueden ser apreciados desde una visión económica tales como el impacto que la normativa tendrá en la práctica, sus consecuencias reales en la esfera social y la predisposición al cumplimiento por parte del sujeto pasivo quien actúa en base a incentivos.

En el caso particular del sistema jurídico ecuatoriano se puede afirmar que el legislador en efecto tiene esta concepción errada descrita en el párrafo anterior y esto lo lleva a confundir eficiencia normativa con formalidad en la creación de la norma, evidentemente esto aumenta los costos de transacción y se debilita el sistema jurídico que se hace menos atractivo ante el mercado global al presentar síntomas de la falta de seguridad jurídica tales como normas poco claras, ambiguas, redundantes, que frecuentemente confunden instituciones legales y que por ello no permiten conocer las consecuencias futuras de los actos y contratos que se realizan.

Una de las causas elevados costos de transacción en el ordenamiento jurídico ecuatoriano que la teoría económica ha logrado identificar es la falta de concordancia que existe entre las reglas de juegos formales e informales, esto significa que existe desconexión de la ley y la realidad que intenta regular.

"Dentro de la teoría económica de la regulación las reglas de juego informales son productos idiosincráticos básicamente complejos y evolutivos que poseen una esencia dependiendo de factores culturales y sociales" (Súmar, 2018, p.33). Precisamente esto se refiere al funcionamiento tradicional de una sociedad y sirve como parámetro en la elaboración de legislación el cual ayudará a evaluar la legitimidad de una regla de juego formal que consiste simplemente en la norma meramente prescriptiva.

Estas leyes meramente prescriptivas pueden resultar especialmente perjudiciales al tráfico jurídico si no consideran la manera en la que funciona la sociedad en la práctica (reglas 
de juego informales) y la consecuencia directa de aquello es que la regla formal no gozará de la legitimidad necesaria debido a que entre los sujetos receptores no se generará una sensación de "justicia" o "razonabilidad".

Cuando las reglas formales son percibidas como ilegítimas aumenta considerablemente el riesgo de incumplimiento por parte de la población, para hacer un símil se puede decir que la norma al estar desprovista de una "base firme" sobre la cual pueda asentarse se ve desobedecida, no por el hecho exclusivo de la voluntad del receptor de ignorarla, sino por la imposibilidad de la norma de encajar en el plano fáctico.

Por lo expuesto anteriormente se puede afirmar que la norma requiere ser concordante a la realidad y tener la capacidad de adaptarse a ella, esto se conoce como elasticidad institucional que según Sumar (2018) tiene como característica la facilidad de la institución formal en ser suplantada por una institución informal y viceversa.

Inclusive, desde la teoría tradicional del derecho se conoce que la norma no puede estar en discordancia con la realidad, es preciso en este punto recordar la teoría de la tridimensionalidad del Derecho que ve a la ciencia jurídica como una convergencia de tres dimensiones: Hecho, valor y norma las mismas que se encuentran profundamente interrelacionadas en una codependencia casi vital y, en consecuencia, si falta alguno de estos elementos el derecho simplemente no existe. (Cano-Nava, 2011, p. 211).

La legislación de Propiedad Intelectual ecuatoriana ha recibido críticas desde distintos sectores por presentar varios de los problemas descritos, sin embargo, es importante resaltar que el ordenamiento jurídico funciona como un todo y para encontrar los motivos que impiden capitalizar los beneficios de la Propiedad Intelectual resulta preciso indicar las problemáticas globales del mismo, aquellas que hacen que en Ecuador los negocios sean muy costosos y se tornen lentos y difíciles de concluir a consecuencia de la ineficiencia del marco normativo, esto nos lleva a otro problema relacionado con lo abordado en líneas anteriores: La sobrerregulación. 
La sobrerregulación es otro de los grandes problemas de nuestra legislación y es responsable de aumentar costos de transacción en las relaciones contractuales, así como en un eventual litigio judicial; en materia de activos de Propiedad Intelectual este problema resulta especialmente preocupante ya que en un mundo globalizado en donde dichos activos tienen un protagonismo cada vez mayor se requiere es celeridad en los negocios y agilidad en la solución de controversias.

Hoy en día, es impensable que en ciertos Estados como el Ecuador sea tan complido registrar una marca u obtener una patente de invención, La burocracia y la ineficiente regulación hacen que realizar estos trámites sea un proceso lento y tortuoso y con costos excesivamente altos.

La sobrerregulación es un concepto utilizado para definir la problemática que surge a partir de la existencia de leyes excesivas que tornan complejo al marco jurídico (Súmar, 2018, p. 75).

Quizá por influencia de su tradición jurídica romana en los países de América Latina es muy común expedir una ley para todo, sin embargo, si bien hay ámbitos de la vida social que merecen la protección y tutela de la ley es necesario realizar una delimitación de aquellos aspectos que deben ser regulados y separarlos de aquellos que no requieren la intervención de la legislación.

Es necesario que la ley no intervenga en puntos donde resulta innecesaria y no hace más que entorpecer procesos que se producen de forma natural en la práctica y específicamente en el mercado; es común observar que en los ordenamientos jurídicos de las economías menos desarrolladas incluso se regulan practicas consuetudinarias como la costumbre mercantil la cual se produce por naturaleza, al ocurrir aquello se aumenta los costos de transacción ya que se desnaturaliza su sentido y alcance e impidiendo incluso su relación con otros sistemas costumbristas como el Common Law.

La costumbre es un sistema que subsiste por sí mismo, se deriva de la interacción humana y da lineamientos éticos y prácticos a las relaciones jurídicas por lo que evidentemente no 
requiere de regulación por parte de normativa alguna que, como máximo, se puede limitar a reconocerla. (Beade, 2016, p.156).

La sobrerregulación también puede dar paso a problemas mucho más graves como la corrupción ya que el Estado pierde capacidad de acción y hace que sus propias instituciones legitimen este tipo de prácticas anti éticas ya que en un escenario regulatorio donde prima la ambigüedad y vaguedad de la norma y las múltiples interpretaciones se hacen comunes, incluso entre quienes ostentan el poder ponen en duda la autoridad de la ley y, por ello, el mismo Estado de Derecho.

Por este motivo, en materia de gestión de la Propiedad Intelectual se hace necesaria la existencia de normas claras y simplificadas que permitan que la administración de los derechos intelectuales sea eficiente, con trámites simplificados y a costos mínimos.

El ordenamiento jurídico ecuatoriano junto a sus elevados costos de transacción en las relaciones jurídico económicas de los ciudadanos desincentiva la consecución de negocios bajo un escenario formal y esto conlleva a un aumento de la informalidad al ser un campo de acción más cercano a las reglas de juego naturales de la sociedad y por ende más accesible y hasta útil para quien intenta entrar desde cero al mercado con un producto o servicio basado en un activo de Propiedad Intelectual.

La variabilidad de la regulación se suma como otro factor que se traduce en inseguridad jurídica, este es un problema que trae severas dificultades para que el país sea atractivo para empresas extranjeras que deseen explotar sus activos intangibles en el país y esto perjudica seriamente al proceso de transferencia de tecnología.

Por último, es importante mencionar que las políticas públicas en materia no suelen ser las más adecuadas y resultan perniciosas en muchasocasiones; las decisionesgubernamentales y sus repercusiones en el goce de los derechos de propiedad intelectual deben ser analizados de forma integral para evitar 
problemas tales como el abuso de licencias obligatorias, una práctica que evidentemente no genera incentivos para explotar activos de Propiedad Intelectual en el país.

La creación de políticas públicas y legislación es una tarea dificultosa ya que se requiere un equipo de trabajo multidisciplinario capaz de no incurrir en algún sesgo sin regresar la vista a los hechos y realidades que se producen en la sociedad (Franco, 2012, p. 3).

\section{OTROS PROBLEMAS DEL ÁMBITO ECUATORIANO QUE LIMITAN LA GENERACIÓN DE RIQUEZA EN BASE A LA PROPIEDAD INTELECTUAL Y SUS POTENCIALES SOLUCIONES.}

En este acápite se procederá a abordar algunas cuestiones adicionales que impiden obtener provecho de los activos intangible de Propiedad Intelectual de forma eficiente y se plantearán algunos mecanismos como posibles soluciones a esta problemática.

En primer lugar, resulta pertinente resaltar ciertos problemas de la legislación mercantil que se constituye en sí misma como el régimen encargado de garantizar el flujo de capitales y el normal desenvolvimiento del comercio en el Ecuador.

Es importante mencionar la reciente expedición del nuevo Código de Comercio en mayo el 2019 que promete ser una ley capaz de actualizar la materia mercantil y las antiguas instituciones contenidas en el código de comercio anterior que no habían tenido una evolución legislativa desde la época en la que el General Eloy Alfaro ocupó la Presidencia de la República.

Pese a que la nueva legislación mercantil ha incluido temas novedosos como el comercio electrónico y algunas otras nociones comerciales que hoy en día se utilizan en las economías más desarrolladas del mundo se debe señalar que estas actualizaciones no se han traducido necesariamente en una disminución en los costos de transacción, el código de comercio del 2019 también acarrea algunos problemas como la 
sobrerregulación y la confusión de instituciones.

Respecto a este último punto se puede hablar de forma abundante, el legislador tiende a confundir instituciones en varios cuerpos normativos de la legislación ecuatoriana, sin embargo, en este punto es pertinente resaltar que en la legislación mercantil esto puede suponer un riesgo precisamente porque pueden aumentar considerablemente los costos de transacción en las relaciones comerciales donde inclusive se realizan transacciones con activos de Propiedad Intelectual.

Un ejemplo de una institución que ha sido confundida por el legislador y que tiende a aumentar los costos de transacción en cualquier negocio es la prestación irrisoria que se define en el artículo 217 del código de comercio como aquella prestación que es ínfima, inequitativa equivalente o desequilibrada en relación a la prestación del otro contratante.

Esta institución es utilizada y de hecho resulta muy útil en contratos de naturaleza civil sin embargo es un error haberla aplicado en la legislación mercantil, sin embargo, esto es precisamente lo que ha hecho el nuevo código de comercio en su artículo 217 donde enuncia dos escenarios en los cuales se puede constituir una prestación en irrisoria:

a) Que la otra parte se haya aprovechado injustificadamente de la dependencia, aflicción económica o necesidades apremiantes de la otra parte, o de su falta de previsión, ignorancia, inexperiencia o falta de habilidad en la negociación.

b) La naturaleza y finalidad del contrato.

(Código de Comercio, 2019, Art.217)

Aplicar la prestación irrisoria en el ámbito mercantil es un error ya que se trata de una institución pensada para proteger a la contraparte que no posee experiencia en negociación dentro de un negocio jurídico, sin embargo, en el campo del Derecho comercial es necesario que exista un saludable flujo de capitales y, sobre todo, que los negocios se realicen con agilidad. 
La ley debe abstenerse se intervenir estos escenarios donde existen otros mecanismos con los que el comerciante se puede blindar, además, en este caso específico la legislación comercial debe dar por hecho que el comerciante es un agente que utiliza el mercado de forma regular y por ende cuanta con la experiencia necesaria; asumir la postura contraria aumentaría los costos de transacción y el riesgo de incumplimiento en los contratos.

En efecto, una de las consecuencias adversas que puede tener la prestación irrisoria en temas comerciales ocurriría, por ejemplo, si en una relación contractual esta es utilizada por uno de los contratantes como una forma "legal" de incumplir el contrato. El sujeto en cuestión perfectamente puede alegar que por alguno de los escenarios mencionados en el artículo 217 del código de comercio que justifiquen que la prestación ha resultado ínfima, inequitativa equivalente o desequilibrada.

A posteriori la inexistencia de prestación irrisoria es complicada de probar y por ello el riesgo en los negocios se ve incrementado lo que obliga a los abogados encargados de la elaboración de contratos a recurrir a elementos adicionales y soluciones bajas para proteger el negocio jurídico como por ejemplo hacer una declaración por escrito en el contrato de que los contratantes tienen experticia en el Comercio, sin embargo, con este tipo de soluciones se pierde la esencia pragmática del contrato y pasa a ser una mera arma legal que no cumple con su finalidad de instrumento facilitador de negocios.

En materia de Propiedad Intelectual los problemas normativos como estos pueden incrementar el litigio y como apunta la solución de controversias en este ámbito no puede limitarse a la judicialización de todas ellas, por su naturaleza esto representaría un coste económico y social importante y perjudicial para capitalizar los beneficios de la Propiedad Intelectual (Lipszyc, 2004, p. 53).

Un problema adicional que está relacionado a lo anterior es la presencia de barreras burocráticas excesivas o irrazonables en el ámbito de gestión de derechos intelectuales. 
Una barrera burocrática es una limitación que la administración impone para que el derecho de los privados a participar del mercado no sea ilimitado y el Estado pueda tener cierto nivel de control respecto a las actividades que se realizan en el mercado. Las barreras burocráticas por sí mismas no son perjudiciales para el mercado, el problema surge cuando estas desalientan la inversión y restringen el acceso al mercado (Baca, 2009, p.78).

En el Ecuador el tema de eliminación de barreras burocráticas no tiene un desarrollo tan vertiginoso como el que ha tenido en países vecinos que poseen una economía mucho más liberalizada como en el caso de Perú, sin embargo, este tema debería ser de gran preocupación en el ámbito nacional en donde restricciones irrazonables de entrada y salida del mercado se constituyen en un obstáculo para la generación de capital.

A lo largo del punto dos y tres de este artículo se ha expuesto una serie de inconvenientes que dificultan la explotación de activos intangibles de Propiedad Intelectual y se ha señalado a la legislación en sus diversos ámbitos (Administrativo, Mercantil, etc.) como su origen inmediato.

En este sentido, para superar estos problemas será necesario buscar mecanismos para que la legislación flexibilice el acceso al mercado y genere emprendimiento e inversión, este no es un trabajo sencillo y requerirá de la aplicación de un programa de mejora regulatoria que tenga como eje la identificación de principios e instituciones confundidos por el legislador y que tienden a aumentar los costos de transacción.

Al mencionar la mejora regulatoria se hace referencia a identificar los factores que hacen ineficiente a la legislación a fin de someterla a un programa de análisis en el cual, bajo principios económicos se buscará optimizarla, hay que resaltar que este examen por sí mismo tienen un carácter continuo en el tiempo (Sumar, 2018, p.37). 
Este proceso de cambio en la regulación, así como toda renovación del paradigma de administración estatal implica una profunda revisión del marco jurídico-administrativo.

Esta labor, sin embargo, requerirá de una coordinación entre los diversos actores del sistema, además que parte del éxito radica en la capacidad de encontrar un punto de equilibrio entre la norma formal y las reglas sociales existentes.

\section{CONCLUSIONES}

A lo largo del presente ensayo se ha realizado un análisis acerca de la importancia de la Propiedad Intelectual para el desarrollo humano y los principales dilemas que debe enfrentar para convertirse en un mecanismo de desarrollo en países como el Ecuador.

En primer lugar, se resaltó el papel fundamental de la propiedad intelectual como un campo de la ciencia jurídica que va más allá de su dimensión proteccionista de los derechos de los creadores y pasa a ser un medio para alcanzar el desarrollo debido a su potencial de incentivar al avance de la ciencia y la tecnología.

Además, se mencionaron algunos factores que impiden capitalizar los beneficios de la propiedad intelectual de forma eficiente, se realizó especial énfasis en que dichos problemas encuentran su origen en la calidad del ordenamiento jurídico y las deficiencias que este posee como la sobrerregulación, la mala interpretación de principios e instituciones por parte del legislador, entre otros, que traen como consecuencia directa el aumento de costos de transacción y dificultan el flujo del mercado en materia de Propiedad Intelectual.

Estas problemáticas no serán fáciles de superar al corto plazo pues han estado presentes desde siempre en América Latina, una región donde gran parte de la población tiene serios inconvenientes para acceder al mercado, por este motivo se hace necesaria la aplicación de programas de mejora regulatoria que corrijan estas falencias en la concepción y aplicación práctica 
del Derecho a fin de reducir costos de transacción, disminuir la litigiosidad y generar eficiencia para de esta manera potenciar la dimensión capitalizadora de la Propiedad Intelectual y alcanzar el desarrollo bajo los más altos estándares internacionales.

\section{REFERENCIAS BIBLIOGRÁFICAS}

Arias, X. Caballero, G. (2003). Instituciones, costos de transacción y políticas públicas: un panorama. Revista de economía institucional. 5(8), (pp. 117-146).

Asamblea Nacional del Ecuador. Código de Comercio. [Cod.]. (2019). R. O. 497 de 29 de mayo de 2019.

Baca, M. Saavedra, H. (2012). Competencia de INDECOPI para conocer de barreras burocráticas que limitan la inversión en servicios Públicos e infraestructura de obras públicas. Revista ITA IUS ESTO, 2 (1), (pp. 77-94).

Beade, L. (2016) Observaciones acerca de la relación entre ética y derecho en la metafísica de las costumbres. Ideas y Valores, 65 (162), (pp. 135-160).

Cano-Nava, M. (2011). Modelo epistemológico de la teoría tridimensional del derecho. Convergencia: Revista de ciencias sociales. 18(57), (pp. 209-228).

Diario El País. (17 de noviembre de 2018). Los valores intangibles pesan más que nunca en la cotización total. Diario El País. Recuperado de https://cincodias.elpais.com/cincodia s/2018/10/16/fortunas/1539709258_777544.html

Franco, J. (2012). Diseño de políticas públicas. Ciudad de México, México: Grupo Editorial y de investigación Polaris.

Lipszyc, D. (2004). Nuevos temas de derecho de autor y derechos conexos. Buenos Aires, Argentina: Editorial Zabalía. 
Londoño, M. Restrepo, L. (2013). Patentes: Herramientas de Innovación. Medellín, Colombia: Editorial Universidad de Medellín. (pp. 25-45.)

Míguez, P. (2018) La propiedad intelectual y la mercantilización forzada del conocimiento. Universitas, Revista de Ciencias Sociales y Humanas, 4 (29), (p. 43-65).

Mutter, K. (2006). Propiedad intelectual y desarrollo en Colombia. Estudios Socio-Jurídicos, 8(2), (pp. 85-101).

Plata, L. (2007). Introducción al análisis económico de los derechos de autor. Revista de Derecho. (pp. 284-299).

Robayo, B. (2017). El enfoque adecuado de la propiedad intelectual desde un país en desarrollo. Iuris Dictio, 19(1), (pp. 261-268).

Rozanski, F. (2003). El valor de la propiedad intelectual en los países en desarrollo. Asociación Interciencia, 28(2), (pp. 105-110).

Súmar, O. (2018). Más Allá del análisis económico de la regulación. Quito, Ecuador: Centro de Publicaciones PUCE. (p. 2037).

Recibido: $21 / 11 / 2019$

Aprobado: 12/06/2020

René Antonio Gálvez Delgado: Investigador jurídico

independiente

Correo electrónico: renegalvez627@gmail.com

Ciudad: Quito

País: Ecuador 
